\title{
New Genic agents to the Treatment of Dyslipidemia
}

\author{
Raffaele La Porta ${ }^{1}$, Giovanni Granata ${ }^{2}$, Francesco Ferrara ${ }^{3}$, and Antonio Vitiello ${ }^{4}$ \\ ${ }^{1}$ ASUR Marche \\ ${ }^{2}$ Local Health Authority Salerno \\ ${ }^{3}$ USL 1 Umbria \\ ${ }^{4}$ Azienda Unità Sanitaria Locale Umbria 1
}

April 28, 2020

\begin{abstract}
Several studies have shown a high correlation between concentration and development of low-density lipoprotein cholesterol (LDL-C)and the evolution of atherosclerosis and cardiovascular disease. Therefore, the reduction of LDL-C levels through lifestyle modification and/or pharmacological interventions has universally shown a decrease in cardiovascular events and mortality. In most cases, elevated blood lipid levels may be caused by alterations in certain genes encoding proteins involved in LDL metabolism, such as those associated with loss of function of the LDLr receptor gene, loss of function of the apoB gene or increased function of a PCSK9 protein .Family hypercholesterolemia is a hereditary disease in which a genetic alteration causes an increase in blood cholesterol. Therapy is based on dietary control and drugs such as statins, ezetimibe or PCSK9 inhibitory monoclonal antibodies. An important scientific breakthrough in recent years is the ability to identify the genetic basis of diseases and possibly correct the defective gene by interfering with small interfering RNA (siRNA) or antisense oligonucleotides (ASO). The technologies of antisense oligonucleotides (ASOs) and small interfering RNAs (siRNAs) have also been developed for the treatment of hypercholesterolemia with the aim of controlling the expression of specific genes that play key roles in lipid metabolism. Anti-sense oligonucleotides have been developed to target apolipoprotein B, the main structural protein of VLDL,LDL and chylomicrons, apolipoproteinCIII or angiopoietin 3, both of which play a role in the regulation of triglyceridesorapo(a). The siRNA approach works on the expression of PCSK9, a key modulator in LDL receptor catabolism. The purpose of this review is to present and discuss current clinical and scientific data on therapeutic evidence for new gene therapies in the treatment of hypercholesterolemia.
\end{abstract}

\section{Hosted file}

New Genic agents to the Treatment of Dyslipidemia 2.pdf available at https://authorea.com/users/ 310211/articles/441020-new-genic-agents-to-the-treatment-of-dyslipidemia 